\title{
Instrumentos para la evaluación de procesos de capacitación sobre violencia
}

Fausto Garmendia, Eva Miranda, Luzmila Figueroa, Patricia Tello, Rosa Zapata, Jorge Miano, Gerardo Ronceros, Diana Francisco

Programa Permanente de Capacitación para la Atención Integral de Victimas de Violencia, Facultad de Medicina, UNMSM Efectuado con fondos del Proyecto Multidisciplinario PMl12011, 2011, VRI, UNMSM

Objetivos: Validar 3 instrumentos de evaluación de procesos de capacitación sobre violencia.

Diseño: Análisis descriptivo, observacional, prospectivo del desempeño laboral de participantes posterior a su capacitación.

Institución: El Programa Permanente de Capacitación para la Atención Integral de Víctimas de Violencia, Facultad de Medicina, UNMSM, impartió la Diplomatura de Atención Integral en Salud a Víctimas de Violencia y el Curso TOT.

Participantes: Profesionales de salud y profesores universitarios de 12 regiones del país.

Intervenciones: 70 profesionales de salud y profesores universitarios de 12 regiones del país recibieron ambas capacitaciones. Para evaluar el efecto de dicha capacitación, se elaboró 3 instrumentos: a) Cuestionario de Seguimiento a Ex alumnos, validado por grupos focales; permitió recabar información personal, laboral, de acciones prestacionales, preventivo-promocionales, educacionales, investigación y gestión de los participantes; b) La Lista de Cotejo de Evidencias permitió obtener evidencias de las actividades realizadas; y, c) la Ficha de Informe de la Visita a las Regiones permitió establecer el desempeño del participante en el lugar de su trabajo.

Principales medidas de resultados: Desempeño laboral.

Resultados: Con estos instrumentos se obtuvo una valiosa información del desempeño de los participantes después de concluida su capacitación, además de triangular la información para contrastar y reforzar los resultados.

Conclusiones: Estos instrumentos son apropiados para evaluar procesos de capacitación sobre violencia.

Palabras clave: Instrumentos de evaluación, capacitación, violencia.

\section{Conductas sexuales de riesgo. Programa de intervención de enfermería para la prevención del VIH-sida en alumnos ingresantes a la Facultad de Medicina, en el año 2010}

Edna Ramírez, Rudi Loli, Ridberth Ramírez, Blanca Fuentes

Facultad de Medicina, UNMSM

Objetivos: Demostrar la efectividad del Programa de intervención de enfermería en el incremento de los conocimientos sobre prevención de ITS y VIH-sida.

Diseño: Cuasi experimental, con un solo grupo, antes y después.

Institución: Facultad de Medicina, UNMSM.

Participantes: Estudiantes ingresantes 2010.

Intervenciones: En una muestra de 285 ingresantes 2010, se aplicó un cuestionario educativo estructurado, antes y después del programa de intervención de enfermería.

Principales medidas de resultados: Evaluación vigesimal.

Resultados: El puntaje promedio global de los conocimiento sobre prevención de ITS y VIH-sida. Antes del programa fue de 12 puntos: en Medicina 12,9, Enfermería 12,8, Obstetricia 12, Tecnología Médica 11,3 y Nutrición 10,4. Después de la intervención, el puntaje promedio de conocimiento en los estudiantes de la Facultad de Medicina fue 15,7; en Medicina y Enfermería 17,1, en Obstetricia 15,1, en Tecnología Médica 14,6 y Nutrición 13,7.

Conclusiones: Existió correlación alta entre la puntuación sobre los conocimientos de prevención de ITS y VIH-sida en los ingresantes de las 5 escuelas, antes y después del programa de intervención ( $r$ mayor 0,8 ). Los conocimientos se incrementaron significativamente en 3,7 puntos; el programa de intervención fue efectivo.

Palabras clave: Conductas sexuales de riesgo, intervención de enfermería, alumnos ingresantes. 